\title{
On the construction of pseudo-hermitian quantum system with a pre-determined metric in the Hilbert space
}

\author{
Pijush K. Ghosh* \\ Department of Physics, Siksha-Bhavana, \\ Visva-Bharati University, \\ Santiniketan, PIN 731 235, India.
}

\begin{abstract}
A class of pseudo-hermitian quantum system with an explicit form of the positive-definite metric in the Hilbert space is presented. The general method involves a realization of the basic canonical commutation relations defining the quantum system in terms of operators those are hermitian with respect to a pre-determined positive definite metric in the Hilbert space. Appropriate combinations of these operators result in a large number of pseudo-hermitian quantum systems admitting entirely real spectra and unitary time evolution. The examples considered include simple harmonic oscillators with complex angular frequencies, Stark(Zeeman) effect with non-hermitian interaction, non-hermitian general quadratic form of $N$ boson(fermion) operators, symmetric and asymmetric XXZ spin-chain in complex magnetic field, non-hermitian Haldane-Shastry spin-chain and LipkinMeshkov-Glick model.
\end{abstract}

*Electronic address: pijushkanti.ghosh@visva-bharati.ac.in 


\section{INTRODUCTION}

In the standard quantum physics, an operator that is equal to its own complex-conjugate transpose is defined as hermitian. Operators not satisfying the above criteria are termed as non-hermitian and have been used extensively to stimulate dissipative quantum processes. However, the discovery [1-5] of a class of such non-hermitian Hamiltonian admitting entirely real spectra with unitary time evolution has given a scope to review this standard practice and to broaden our understanding of quantum physics $[1-22]$. The reality of the entire spectra is related to an underlying unbroken combined $\operatorname{Parity}(\mathcal{P})$ and $\operatorname{Time-reversal}(\mathcal{T})$ symmetry and/or pseudo-hermiticity of the non-hermitian Hamiltonian with a positivedefinite metric in the Hilbert space $1-4]$. Apart from a very few known examples, one of the major technical difficulties in the study of $\mathcal{P} \mathcal{T}$ symmetric and/or pseudo-hermitian quantum physics is to find the appropriate basis with respect to which the non-hermitian Hamiltonian becomes hermitian. It may be mentioned here that the description of a pseudo-hermitian Hamiltonian is incomplete in absence of an explicit knowledge of the metric in the Hilbert space, since neither the completeness of states nor the unitarity can be guaranteed. There are known methods based on spectral decomposition[13, 16], perturbation theory [14, 17], Moyal product[15], group theory [10] etc. to find exact or approximate form of the metric in the Hilbert space of a given $\mathcal{P} \mathcal{T}$ symmetric or pseudo-hermitian Hamiltonian. However, the list is still not exhaustive and there are always scopes for introducing alternative methods and beautiful models based on inherent simplicity and physical relevance.

The purpose of this paper is to present a class of pseudo-hermitian Hamiltonian with an explicit form of the metric in the Hilbert space. The examples include simple harmonic oscillators with complex angular frequencies, Stark(Zeeman) effect with non-hermitian interaction, non-hermitian general quadratic form of $N$ boson(fermion) operators, $X X Z$ spin-chains with complex magnetic field, non-hermitian Haldane-Shastry spin-chain[23] and Lipkin-Meshkov-Glick[24] model. A non-hermitian asymmetric XXZ spin Hamiltonian that is generally used as the time-evolution operator of certain reaction-diffusion processes and 
growth phenomenon is shown to be pseudo-hermitian and may thus be used to describe nondissipative processes by using a modified inner product in the Hilbert space. The approach followed in this paper is the following. The basic canonical commutation relations defining a quantum system are realized in terms of operators those are hermitian with respect to a pre-determined positive-definite metric $\eta_{+}$in the Hilbert space. Consequently, any Hamiltonian that is constructed using appropriate combination of these operators is hermitian with respect to $\eta_{+}$. However, in general, the same Hamiltonian may not be hermitian with respect to the standard Dirac-hermiticity condition, thereby giving rise to a pseudo-hermitian Hamiltonian.

The plan of this paper is the following. In the beginning of the next section, known results on pseudo-hermitian operators[2 4] are reviewed. Thereafter, a general prescription to construct pseudo-hermitian quantum system with a pre-determined metric is presented. In Sec. III, examples of single particle pseudo-hermitian quantum systems are given. A twodimensional pseudo-hermitian simple harmonic oscillator, Stark and Zeeman effect with nonhermitian interaction are discussed in sections III.A, III.B and III.C, respectively. Sec. IV contains examples of many particle pseudo-hermitian quantum systems. In particular, general quadratic forms of boson and fermion operators are discussed in sections IV.A and IV.B, respectively. In Sec. IV.A, Schwinger's oscillator model of angular momentum is generalized to pseudo-hermitian operators and a non-hermitian version of the Lipkin-Meshkov-Glick model is introduced. Pseudo-hermitian XXZ spin-chain and Haldane-Shastry spin-chain are presented in sections IV.C. Finally, the findings of this paper are summarized with possible implications in section $\mathrm{V}$.

\section{FORMALISM}

An operator $\hat{A}$ that is related to its adjoint $\hat{A}^{\dagger}$ through a similarity transformation is known as pseudo-hermitian [2, 3],

$$
\hat{A}^{\dagger}=\eta \hat{A} \eta^{-1}
$$


In general, the operator $\eta$ is not unique for a given pseudo-hermitian operator $\hat{A}$. Among all possible forms of $\eta$, a positive-definite $\eta_{+}$is chosen to define a modified inner product in the Hilbert space of $\hat{A}$ as follows:

$$
\langle\langle., .\rangle\rangle_{\eta_{+}}:=\left\langle., \eta_{+} \cdot\right\rangle .
$$

The operator $\eta_{+}$plays the role of a metric in the Hilbert space and the standard inner product $\langle.,$.$\rangle is obtained in the limit when \eta_{+}$is replaced by the Identity operator. The Hilbert space that is endowed with the metric $\eta_{+}$with the modified inner product (2) is denoted as $\mathcal{H}_{\eta_{+}}$. On the other hand, the Hilbert space that is endowed with the standard inner product $\langle.,$.$\rangle is denoted as \mathcal{H}_{D}$. The subscript $D$ indicates that the Dirac-hermiticity condition is used in this Hilbert space. The pseudo-hermitian operator $\hat{A}$ is hermitian in the Hilbert space $\mathcal{H}_{\eta_{+}}$. In an alternative formulation of the same problem, $\hat{A}$ can be mapped to an operator $\hat{\mathcal{A}}$ that is hermitian in $\mathcal{H}_{D}$. In particular,

$$
\hat{\mathcal{A}}=\rho \hat{A} \rho^{-1}, \quad \rho:=\sqrt{\eta_{+}} .
$$

The operator $\hat{\mathcal{A}}$ satisfying the above relation is known as quasi-hermitian[4]]. It may be noted that the Hilbert spaces of $\hat{A}$ and $\hat{\mathcal{A}}$ are different. Corresponding to a hermitian operator $\hat{\mathcal{B}}$ in the Hilbert space $\mathcal{H}_{D}$ of $\hat{\mathcal{A}}$, a hermitian operator $\hat{B}$ in the Hilbert space $\mathcal{H}_{\eta_{+}}$of $\hat{A}$ can be defined as[2],

$$
\hat{B}=\rho^{-1} \hat{\mathcal{B}} \rho .
$$

The above relation is important for the identification of physical observables in the Hilbert space $\mathcal{H}_{\eta_{+}}$of $\hat{A}$. An interesting consequence of Eq. (4I) is that a set of operators $\hat{\mathcal{B}}_{i}$ obey the same canonical commutation relations as those satisfied by the corresponding set of operators $\hat{B}_{i}$ and the vice verse.

The coordinates and the conjugate momenta which are hermitian in the Hilbert space $\mathcal{H}_{D}$ that is endowed with the standard inner product $\langle.,$.$\rangle are denoted as (x, y, z)$ and $\left(p_{x}, p_{y}, p_{z}\right)$, respectively. In the coordinate space representation, the momenta and the orbital angular momentum operators have the following standard form:

$$
p_{x}=-i \frac{\partial}{\partial x}, p_{y}=-i \frac{\partial}{\partial y}, p_{z}=-i \frac{\partial}{\partial z}, \mathcal{L}_{x}=y p_{z}-z p_{y}, \mathcal{L}_{y}=z p_{x}-x p_{z}, \mathcal{L}_{z}=x p_{y}-y p_{x} .
$$


A positive-definite metric $\eta_{+}$in the Hilbert space $\mathcal{H}_{\eta_{+}}$may now be considered:

$$
\eta_{+}:=e^{-2 \gamma \mathcal{L}_{z}}, \quad \gamma \in R
$$

Metric of the form (6) has been considered previously in the study of a variety of pseudohermitian quantum mechanical systems [2, 7, 8, 20]. The operators $(x, y)$ and $\left(p_{x}, p_{y}\right)$ are no more hermitian in the Hilbert space $\mathcal{H}_{\eta_{+}}$. A new set of canonical conjugate operators those are hermitian in the Hilbert space $\mathcal{H}_{\eta_{+}}$may be introduced as follows:

$$
\begin{aligned}
& X=x \cosh w+i y \sinh w, \quad Y=-i x \sinh w+y \cosh w, \quad Z=z \\
& P_{X}=p_{x} \cosh w+i p_{y} \sinh w, P_{Y}=-i p_{x} \sinh w+p_{y} \cosh w, P_{Z}=p_{z} \\
& w \equiv \gamma+i \xi, \quad \xi \in R .
\end{aligned}
$$

The transformation matrix $M$,

$$
M \equiv\left(\begin{array}{cc}
\cosh w & i \sinh w \\
-i \sinh w & \cosh w
\end{array}\right)
$$

relating $(X, Y)$ to $(x, y)$ and $\left(P_{X}, P_{y}\right)$ to $\left(p_{x}, p_{y}\right)$, has appeared previously in the study of two-level pseudo-hermitian quantum systems [2, 20]. Note that the length remains invariant under the transformation defined by Eq. (7), i.e. $R^{2} \equiv X^{2}+Y^{2}+Z^{2}=r^{2} \equiv x^{2}+y^{2}+z^{2}$. The same is true for the total momentum square, $P^{2} \equiv P_{X}^{2}+P_{Y}^{2}+P_{Z}^{2}=p^{2} \equiv p_{x}^{2}+p_{y}^{2}+p_{z}^{2}$. The operators $\left(X, Y, P_{X}, P_{Y}\right)$ defined by Eq. (77) are not hermitian with respect to the standard inner product for $\gamma \neq 0$. The angular momentum operators,

$$
L_{X}:=Y P_{Z}-Z P_{Y}, L_{Y}:=Z P_{X}-X P_{Z}, L_{Z}:=X P_{Y}-Y P_{X}, L^{2}:=L_{X}^{2}+L_{Y}^{2}+L_{Z}^{2}
$$

are related to $\mathcal{L}_{x}, \mathcal{L}_{y}, \mathcal{L}_{z}$ and $\mathcal{L}^{2}:=\mathcal{L}_{x}^{2}+\mathcal{L}_{y}^{2}+\mathcal{L}_{z}^{2}$ through the equations:

$$
\begin{aligned}
& L_{X}=\cosh w \mathcal{L}_{x}+i \sinh w \mathcal{L}_{y} \\
& L_{Y}=-i \sinh w \mathcal{L}_{x}+\cosh w \mathcal{L}_{y} \\
& L_{Z}=\mathcal{L}_{z}, \quad L^{2}=\mathcal{L}^{2} .
\end{aligned}
$$

For $\gamma \neq 0$, the operators $L_{X}$ and $L_{Y}$ are not hermitian with respect to the standard inner product, but, are hermitian with respect to the modified inner product. The operators 
$\left(Z, P_{Z}, L_{Z}, R, P, L^{2}\right)$ or equivalently $\left(z, p_{z}, \mathcal{L}_{z}, r, p, \mathcal{L}^{2}\right)$ are hermitian in $\mathcal{H}_{\mathcal{D}}$ as well as in $\mathcal{H}_{\eta_{+}}$. The operators $X, Y, P_{X}, P_{Y}, L_{X}, L_{Y}$ are hermitian in $\mathcal{H}_{\eta_{+}}$and the quasi-hermiticity[4] of these operators may be checked as follows:

$$
\begin{aligned}
& x=(U \rho) X(U \rho)^{-1}, y=(U \rho) Y(U \rho)^{-1}, p_{x, y}=(U \rho) P_{X, Y}(U \rho)^{-1}, \mathcal{L}_{x, y}=(U \rho) L_{X, Y}(U \rho)^{-1}, \\
& U:=e^{-i \xi \mathcal{L}_{z}}, \quad U^{\dagger}=U^{-1}=e^{i \xi \mathcal{L}_{z}},
\end{aligned}
$$

where $t^{*}$ denotes complex conjugation of $t$. The property of quasi-hermiticity of the operators $X, Y, P_{X, Y}, L_{X, Y}$ may be shown without the use of the unitary operator $U$. For example,

$$
\begin{aligned}
& \rho X \rho^{-1}=x \cos \xi-y \sin \xi \\
& \rho Y \rho^{-1}=x \sin \xi+y \cos \xi .
\end{aligned}
$$

Similar relations between $P_{X, Y}\left(L_{X, Y}\right)$ and $p_{x, y}\left(\mathcal{L}_{x, y}\right)$ also exist. The unitary operation using $U$ has been performed in Eq. (11) to rotate away insignificant terms in the expressions of equivalent hermitian operators in $\mathcal{H}_{D}$. For $\gamma=0, \eta_{+}$reduces to the Identity operator and hence, both the Hilbert spaces become identical. If we further fix $\xi=0,\left(X, Y, Z, P_{X}, P_{Y}, P_{Z}\right)$ become identical with $\left(x, y, z, p_{x}, p_{y}, p_{z}\right)$.

The metric operator $\eta_{+}$is hermitian. If the factor $-2 \gamma$ is replaced by a purely imaginary number $-i \phi, \phi \in(0,2 \pi)$, it would correspond to a rotation by an angle $\phi$ around the $z$ axis. The operator $\eta_{+}$given in Eq. (6) may thus be referred to as generating a 'complex rotation' around the $z$-axis by an amount equal to $2 \gamma$. The metric operator corresponding to a 'complex rotation' around $x$ - or $y$ - axis or even around any arbitrary three dimensional unit vector $\hat{n}$ may be constructed: $\hat{\eta}_{+}:=e^{-2 \gamma \hat{n} \cdot \overrightarrow{\mathcal{L}}}$, where $\overrightarrow{\mathcal{L}}$ is the three dimensional angular momentum operator and the results of this paper may be generalized. In this paper, however, discussion is restricted to the metric $\eta_{+}$given by Eq. (6), unless mentioned otherwise.

Suitable combinations of the operators $X, Y, Z, P_{X}, P_{Y}, P_{Z}$ would result in a very large number of pseudo-hermitian quantum systems, since these operators are pseudo-hermitian by construction. A general non-relativistic pseudo-hermitian quantum system in an external static electric and magnetic field is described by a Hamiltonian of the form [2, 17, 18],

$$
H=\frac{1}{2 m}(\vec{P}-e \vec{A}(X, Y, Z))^{2}+V(X, Y, Z)+e A_{0}(X, Y, Z),
$$


where the vector potential $\vec{A}(X, Y, Z)$, the scalar potential $A_{0}(X, Y, Z)$ and the potential $V(X, Y, Z)$ are real functions of their arguments. The constants $m$ and $e$ are the mass and the charge of the particle, respectively. The form of the minimal coupling to the gauge field in the Hamiltonian $H$ is determined by demanding $U(1)$ invariance [17, 18]. Subtleties involving gauge transformation in pseudo-hermitian quantum systems are discussed in Ref. [17, 18]. The reason for a straightforward and form-invariant extension of the minimal gauge coupling principle of a hermitian theory to a pseudo(quasi)-hermitian one could be understood in a simple manner. The Hamiltonian $H$ is hermitian in $\mathcal{H}_{\eta_{+}}$and non-hermitian in $\mathcal{H}_{D}$, when expressed in terms of $\left(x, y, z, p_{x}, p_{y}, p_{z}\right)$. However, it can be mapped to a hermitian Hamiltonian $h$ in $\mathcal{H}_{D}$ through the similarity transformation:

$$
\begin{aligned}
h & =(U \rho) H(U \rho)^{-1} \\
& =\frac{1}{2 m}(\vec{p}-e \vec{A}(x, y, z))^{2}+V(x, y, z)+e A_{0}(x, y, z) .
\end{aligned}
$$

The minimal gauge coupling in $h$ due to $U(1)$ invariance has the standard form. Thus, the form of the coupling to gauge field in $H$ is justified, if the standard minimal coupling principle due to $U(1)$ gauge invariance is to be maintained for the equivalent hermitian Hamiltonian $h$. The coulomb-gauge condition in $\mathcal{H}_{D}$ is $\vec{p} \cdot \vec{A}(x, y, z)=0$ and in $\mathcal{H}_{\eta_{+}}$:

$$
\vec{P} \cdot \vec{A}(X, Y, Z)=(U \rho)^{-1}(\vec{p} \cdot \vec{A}(x, y, z))(U \rho)=0 .
$$

It may be noted that $H$ and $h$ are isospectral, since they are related to each other through a similarity transformation. However, the eigenfunctions are different. The electromagnetic transition rate between two given states is also identical [17, 18].

The spin degrees of freedom of a particle can also be included in the discussion of nonrelativistic pseudo-hermitian quantum system. To this end, a positive-definite metric in the Hilbert space may be defined as the direct product of $\eta_{+}$and the metric $\zeta_{+}$corresponding to the spin degrees of freedom,

$$
\eta_{+}^{\text {Total }}:=\eta_{+} \otimes \zeta_{+}, \quad \zeta_{+}:=e^{-2 \delta \hat{m} \cdot \overrightarrow{\mathcal{S}}}, \quad \delta \in R,
$$

where $\overrightarrow{\mathcal{S}}$ is the spin operator with components $\mathcal{S}_{x, y, z}$ which are hermitian with respect to the standard inner product and $\hat{m}$ is a unit vector. Hermitian spin operators $T_{X, Y, Z}$ with 
respect to the modified inner product $\langle\langle., .\rangle\rangle_{\zeta_{+}}:=\left\langle., \zeta_{+} \cdot\right\rangle$ may now be constructed using Eq. (4). Restricting the discussion to a simpler case where $\hat{m}$ corresponds to a unit vector along $\mathcal{S}_{z}$, operators $T_{X, Y, Z}$ may be defined as,

$$
\begin{aligned}
& T_{X}:=\cosh \beta \mathcal{S}_{x}+i \sinh \beta \mathcal{S}_{y} \\
& T_{Y}:=-i \sinh \beta \mathcal{S}_{x}+\cosh \beta \mathcal{S}_{y} \\
& T_{Z}:=\mathcal{S}_{z}, \beta \equiv \delta+i \chi, \quad \chi \in R .
\end{aligned}
$$

It may be noted that $\left(T_{Z}, T^{2}\right)$ and $\left(\mathcal{S}_{z}, \mathcal{S}^{2}\right)$ are hermitian with respect to both $\langle.,$.$\rangle as well as$ $\langle\langle., .\rangle\rangle_{\zeta_{+}}$. Spin-orbit interaction of the form $H_{L \mathcal{S}}=f(R) \vec{L} \cdot \vec{T}$, where $f(R)$ is a real function

of $R$, is hermitian with respect to the inner product $\langle\langle., .\rangle\rangle_{\eta_{+}^{T o t a l}}$. Thus, the Hamiltonian $\tilde{H}=H+H_{L \mathcal{S}}$ or its variants involving both spatial as well as spin degrees of freedom are hermitian in the Hilbert space $\mathcal{H}_{\eta_{+}^{\text {Total }}}$ that is endowed with the metric $\eta_{+}^{\text {Total }}$.

Several examples realizing the above formalism are considered in the next few sections. Examples in this paper are chosen based on their simplicity, physical relevance and in some cases exact solvability. The last criteria is very important in the following sense. The positive-definite metric in the Hilbert space can not be calculated exactly for many of the pseudo-hermitian quantum systems known so far. Perturbative and/or numerical methods are used to find approximate form of the metric. Accuracy of these methods may be checked by using an exactly solvable pseudo-hermitian system. The physical relevance of the chosen quantum system is also very important, since experimental realization of the predictions emanating from pseudo-hermitian $/ \mathcal{P} \mathcal{T}$-symmetric quantum mechanics is desirable.

\section{EXAMPLES: SINGLE PARTICLE SYSTEM}

In this section, examples of (i) a two dimensional simple harmonic oscillator with complex angular frequencies, (ii) Stark effect in an external uniform complex electric field and (iii) Zeeman effect with non-hermitian interaction are considered. 


\section{A. Simple Harmonic Oscillator}

$\mathcal{P} \mathcal{T}$ symmetric oscillator in one dimension has been considered in the literature[11], where the hermitian and the non-hermitian Hamiltonian in $\mathcal{H}_{D}$ are related to each other through an imaginary shift of the coordinate. A two dimensional simple harmonic oscillator with complex angular frequencies $\left\{\omega_{1}, \omega_{2}, \omega_{3}\right\}$ that admit entirely real spectra is presented below:

$$
\begin{aligned}
H & =\frac{1}{2 m}\left(p_{x}^{2}+p_{y}^{2}\right)+\frac{m}{2}\left(\omega_{1}^{2} x^{2}+\omega_{2}^{2} y^{2}+\omega_{3}^{2} x y\right) \\
m \omega_{1}^{2} & =k_{1} \cosh ^{2} w-k_{2} \sinh ^{2} w-i k_{3} \cosh w \sinh w \\
m \omega_{2}^{2} & =k_{2} \cosh ^{2} w-k_{1} \sinh ^{2} w+i k_{3} \cosh w \sinh w \\
m \omega_{3}^{2} & =2 i\left(k_{1}-k_{2}\right) \cosh w \sinh w+k_{3}\left(\cosh ^{2} w+\sinh ^{2} w\right),
\end{aligned}
$$

where $\left\{k_{1}, k_{2}, k_{3}, m\right\} \in R$. The non-hermitian Hamiltonian in $\mathcal{H}_{D}$ is related to a hermitian Hamiltonian in $\mathcal{H}_{D}$ through a complex-hyperbolic transformation of the form (7). It is worth mentioning here that simple harmonic oscillators with complex angular frequencies

appear in the description of electromagnetic pulse propagation in a free electron laser [25]. Simple harmonic oscillators with complex angular frequencies have also been studied in the context of squeezed states[26], coherent states[27], tunneling phenomenon in non-hermitian theory[28] and resonant states[29]. In general, the eigenvalues for the above cases are complex and the time-evolution is non-unitary. Within the context of $\mathcal{P} \mathcal{T}$ symmetric theory on a non-commutative space or with a deformed Heisenberg algebra, a simple harmonic oscillator with complex mass and complex angular frequency that admits real spectra within restricted regions of the parameter space has also been studied[30]. The harmonic oscillator Hamiltonian presented in this paper is on the standard Euclidean space and with real mass.

Although $H$ is non-hermitian in $\mathcal{H}_{D}$, it is hermitian in $\mathcal{H}_{\eta_{+}}$. In particular, $H$ can be rewritten as,

$$
H=\frac{1}{2 m}\left(P_{X}^{2}+P_{Y}^{2}\right)+\frac{1}{2}\left(k_{1} X^{2}+k_{2} Y^{2}+k_{3} X Y\right) .
$$

The quasi-hermiticity of $H$ can also be shown by mapping it to $h:=(U \rho) H(U \rho)^{-1}$,

$$
h=\frac{1}{2 m}\left(p_{x}^{2}+p_{y}^{2}\right)+\frac{1}{2}\left(k_{1} x^{2}+k_{2} y^{2}+k_{3} x y\right),
$$


where the operators $U$ and $\rho$ are as defined below:

$$
U:=e^{-i \xi \mathcal{L}_{z}}, \quad \rho:=e^{-\gamma \mathcal{L}_{z}}
$$

The Hamiltonian $h$ is hermitian in $\mathcal{H}_{D}$. The energy eigenvalues of $h$ and hence, of $H$ are determined for the range of the parameters $k_{1,2}>0,4 k_{1} k_{2}>k_{3}^{2}$ as follows:

$$
\begin{aligned}
& E_{n_{x}, n_{y}}=\left(n_{x}+\frac{1}{2}\right) \lambda_{+}+\left(n_{y}+\frac{1}{2}\right) \lambda_{-}, \\
& \lambda_{ \pm} \equiv \frac{1}{2 \sqrt{m}}\left(k_{1}+k_{2} \pm \sqrt{k_{3}^{2}+\left(k_{1}-k_{2}\right)^{2}}\right)^{\frac{1}{2}}, n_{x}, n_{y}=0,1,2, \ldots
\end{aligned}
$$

The corresponding eigenfunctions of $H$ are,

$$
\Psi_{n_{x}, n_{y}}(u, v)=e^{w L_{z}(u, v)} \psi_{n_{x}}(u) \psi_{n_{y}}(v), \quad L_{z}(u, v)=-i\left(u \frac{\partial}{\partial v}-v \frac{\partial}{\partial u}\right)
$$

where $\psi_{n}(u)$ corresponds to $n$th normalized eigenfunction of the standard one dimensional simple harmonic oscillator and the co-ordinates $(u, v)$ are obtained from $(x, y)$ through a rotation on the two-dimensional plane by an angle $\theta$ :

$$
\theta=\frac{1}{2} \tan ^{-1} \frac{k_{3}}{k_{1}-k_{2}}, k_{1} \neq k_{2} ; \quad \theta=\frac{\pi}{4}, k_{1}=k_{2}
$$

For $\lambda_{+} \neq \lambda_{-}$(i.e. $k_{1} \neq k_{2}$ and $k_{3} \neq 0$ ), neither $\psi_{n_{x}}(u) \psi_{n_{y}}(v)$ are eigenfunctions of $L_{z}(u, v)$, nor a basis can be chosen in which simultaneous eigen-functions of $L_{z}(u, v)$ and $h(u, v)$ can be constructed. The following identity can be shown using the properties of the Hermite Polynomials:

$$
\bar{L}_{z}\left[\psi_{n}(u) \psi_{m}(v)\right]=m \psi_{n+1}(u) \psi_{m-1}(v)-n \psi_{n-1}(u) \psi_{m+1}(v), \quad \bar{L}_{z} \equiv i L_{z}
$$

It may be noted that the ground state $\Psi_{0,0}(u, v)=\psi_{0}(u) \psi_{0}(v)$. However, the excited states are determined in terms of an infinite series and a general term in this series will contain expression of the form:

$$
\begin{aligned}
\bar{L}_{z}^{k}\left[\psi_{n}(u) \psi_{m}(v)\right] & =\sum_{i=0}^{\frac{k}{2}}\left[A_{k i} \psi_{n+2 i}(u) \psi_{m-2 i}(v)+B_{k i} \psi_{n-2 i}(u) \psi_{m+2 i}(v)\right], \text { even } k \\
& =\sum_{i=0}^{\frac{k-1}{2}}\left[A_{k i} \psi_{n+2 i+1}(u) \psi_{m-2 i-1}(v)+B_{k i} \psi_{n-2 i-1}(u) \psi_{m+2 i+1}(v)\right], \text { odd } k(26)
\end{aligned}
$$


where the real constants $A_{k i}$ and $B_{k i}$ are determined in terms of $n$ and $m$ for fixed $k$ and $i$. Thus, the wave-function $\Psi_{n_{x}, n_{y}}(u, v)$ is expressed in terms of its arguments in a non-trivial way. It is worth mentioning that the explicit form of $\Psi_{n_{x}, n_{y}}$ in terms of an infinite series is not required to calculate expectation values or matrix elements of any observables in $\mathcal{H}_{\eta_{+}}$. The form of $\Psi_{n_{x}, n_{y}}$, as given in Eq. (23) , is sufficient for this purpose. The factor $e^{w L_{z}}$ cancels out with a similar factor coming from the metric in the Hilbert space. In particular, $\left\langle\left\langle\Psi_{n_{x}^{\prime} n_{y}^{\prime}}|\hat{A}| \Psi_{n_{x} n_{y}}\right\rangle\right\rangle_{\eta_{+}}=\left\langle\psi_{n_{x}^{\prime}}(u) \psi_{n_{y}^{\prime}}(v)|\hat{A}| \psi_{n_{x}}(u) \psi_{n_{y}}(v)\right\rangle$. The orthonormal property of $\psi_{n_{x}}$ together with Eq. (23) can be used to show that $\Psi_{n_{x}, n_{y}}(u, v)$ for all allowed values of the quantum numbers $n_{x}$ and $n_{y}$ constitute a complete set of orthonormal wave-functions in $\mathcal{H}_{\eta_{+}}$.

\section{B. Stark effect}

A non-hermitian Hamiltonian in $\mathcal{H}_{D}$ may be considered as,

$$
H=\frac{p^{2}}{2 m}-\frac{e^{2}}{r}+e \mathcal{E}(x \cosh w+i y \sinh w), \quad \mathcal{E} \in R
$$

which has a similarity with the Hamiltonian describing the Hydrogen atom in an external uniform "static complex electric field" $\overrightarrow{\mathcal{E}}=-\mathcal{E}(\cosh w \hat{i}+\hat{j} i$ sinhw $)$, where $\hat{i}, \hat{j}$ correspond to unit vectors along $x$ and $y$ directions, respectively. It may be noted that although the magnitude of the external electric field is real, the $y$-component of the electric field is purely imaginary. This is the reason to identify $H$ in $\mathcal{H}_{D}$ as describing Stark effect in a "static complex electric field". It is worth mentioning here that with the proper identification of a set of canonical operators, $H$ in $\mathcal{H}_{\eta_{+}}$can be identified as describing Stark effect with real electric field. The possibility of generating non-hermitian interaction terms of $H$ in $\mathcal{H}_{D}$ in the effective description of some hitherto unknown quantum mechanical system which is not subjected to any external electric field also exists. However, in absence of any concrete proposal on how such non-hermitian interaction could be realized in realistic physical systems, the Hamiltonian should be considered as hypothetical one. Nevertheless, the related discussions may be useful to elucidate many technical issues related to pseudo-hermitian 
quantum systems.

The Hamiltonian $H$ can be rewritten as,

$$
H=\frac{P^{2}}{2 m}-\frac{e^{2}}{R}+e \mathcal{E} X
$$

implying that it is hermitian in $\mathcal{H}_{\eta_{+}}$. The equivalent hermitian Hamiltonian in $\mathcal{H}_{D}$,

$$
h:=(U \rho) H(U \rho)^{-1}=\frac{p^{2}}{2 m}-\frac{e^{2}}{r}+e \mathcal{E} x,
$$

can be cast into the standard form,

$$
\tilde{h}:=e^{-i \frac{\pi}{2} \mathcal{L}_{y}} h e^{i \frac{\pi}{2} \mathcal{L}_{y}} h=\frac{p^{2}}{2 m}-\frac{e^{2}}{r}+e \mathcal{E} z,
$$

by a $\frac{\pi}{2}$ rotation around the $y$-axis, where the operators $U$ and $\rho$ are as defined in Eq. (21). The operators $h, \tilde{h}$ and $H$ are isospectral, since they are related to each other through similarity transformations. Following the discussions of Refs. [17, 18], the electromagnetic transition rate between any two states is also identical for $h$ and $H$.

The perturbative analysis of $h(\tilde{h})$ is given in any standard text on quantum mechanics. A perturbative analysis of the pseudo-hermitian $H$ in Eq. (27) may also be carried out directly to obtain the known results. The Hamiltonian $H$ can be rewritten in terms of the unperturbed Hamiltonian $H_{0}$ and the perturbation $H^{\prime}$ as,

$$
H=H_{0}+H^{\prime}, \quad H_{0}=\frac{p^{2}}{2 m}-\frac{e^{2}}{r}, \quad H^{\prime}=e \mathcal{E}(x \cosh w+i y \sinh w) .
$$

The unperturbed Hamiltonian $H_{0}$ is hermitian in $\mathcal{H}_{D}$ and commutes with $\mathcal{L}^{2}$ and $\mathcal{L}_{z}$. A complete set of orthonormal eigenstates of $H_{0}$ with energy $E_{n}$ in $\mathcal{H}_{D}$ are denoted as $\psi_{n l m}$, where $n$ is the principal quantum number, $l$ is the azimuthal quantum number and $m$ is the magnetic quantum number. The principal quantum number $n$ can take any values from the set of positive integers, $l=0,1, \ldots n-1$ and $m=-l,-l+1, \ldots, l-1, l$. The states $\psi_{n l m}$ are simultaneous eigenstates of $H, \mathcal{L}^{2}$ and $\mathcal{L}_{z}$. The perturbing Hamiltonian $H^{\prime}$ is non-hermitian in $\mathcal{H}_{D}$ and in general,

$$
\left\langle\psi_{n l m}\left|H^{\prime}\right| \psi_{n^{\prime} l^{\prime} m^{\prime}}\right\rangle \neq\left\langle\psi_{n^{\prime} l^{\prime} m^{\prime}}\left|H^{\prime}\right| \psi_{n l m}\right\rangle^{*}
$$


which can be checked easily by using the following identities,

$$
\begin{aligned}
& \left\langle\psi_{n l m}\left|\frac{H^{\prime}}{e \mathcal{E}}\right| \psi_{n^{\prime} l^{\prime} m^{\prime}}\right\rangle=\left\langle\psi_{n l m}\left|\rho^{-1} x \rho\right| \psi_{n^{\prime} l^{\prime} m^{\prime}}\right\rangle=e^{\left(m-m^{\prime}\right) \gamma}\left\langle\psi_{n l m}|x| \psi_{n^{\prime} l^{\prime} m^{\prime}}\right\rangle \\
& \left\langle\psi_{n^{\prime} l^{\prime} m^{\prime}}\left|\frac{H^{\prime}}{e \mathcal{E}}\right| \psi_{n l m}\right\rangle=\left\langle\psi_{n^{\prime} l^{\prime} m^{\prime}}\left|\rho^{-1} x \rho\right| \psi_{n l m}\right\rangle=e^{-\left(m-m^{\prime}\right) \gamma}\left\langle\psi_{n^{\prime} l^{\prime} m^{\prime}}|x| \psi_{n l m}\right\rangle .
\end{aligned}
$$

The matrix elements are identical either for $m=m^{\prime}$ or in the hermitian limit $\gamma=0$. The first order correction to the ground state vanishes identically, since $\left\langle\psi_{100}\left|H^{\prime}\right| \psi_{100}\right\rangle=$ $e \mathcal{E}\left\langle\psi_{100}|x| \psi_{100}\right\rangle=0$. The second order correction to the ground state and the first order correction to the first excited state involve product of the matrix elements of the form,

$$
\begin{aligned}
\left\langle\psi_{n l m}\left|H^{\prime}\right| \psi_{n^{\prime} l^{\prime} m^{\prime}}\right\rangle\left\langle\psi_{n^{\prime} l^{\prime} m^{\prime}}\left|H^{\prime}\right| \psi_{n l m}\right\rangle & =(e \mathcal{E})^{2}\left\langle\psi_{n l m}|x| \psi_{n^{\prime} l^{\prime} m^{\prime}}\right\rangle\left\langle\psi_{n^{\prime} l^{\prime} m^{\prime}}|x| \psi_{n l m}\right\rangle \\
& =(e \mathcal{E})^{2}\left|\left\langle\psi_{n l m}|x| \psi_{n^{\prime} l^{\prime} m^{\prime}}\right\rangle\right|^{2}
\end{aligned}
$$

which is real and its value is equivalent to the case when the perturbation is taken as $e \mathcal{E} x$. It is worth recalling at this point that in the perturbative analysis of the equivalent hermitian Hamiltonian $h:=\rho H \rho^{-1}=H_{0}+e \mathcal{E} x$, the perturbing term is indeed given by $e \mathcal{E} x$. Thus, the known results are reproduced. The change in the energy $E_{n}$ of the state $\psi_{n l m}$ due to perturbative corrections at all orders in $H^{\prime}$ higher than the first involves products of matrix elements of the form,

$$
\left\langle\psi_{n l m}\left|H^{\prime}\right| \psi_{n_{k} l_{k} m_{k}}\right\rangle\left\langle\psi_{n_{k} l_{k} m_{k}}\left|H^{\prime}\right| \psi_{n_{k-1} l_{k-1} m_{k-1}}\right\rangle \ldots\left\langle\psi_{n_{2} l_{2} m_{2}}\left|H^{\prime}\right| \psi_{n_{1} l_{1} m_{1}}\right\rangle\left\langle\psi_{n_{1} l_{1} m_{1}}\left|H^{\prime}\right| \psi_{n l m}\right\rangle
$$

where $k \geq 1$ and all the intermediate states $\psi_{n_{k}, l_{k}, m_{k}}, \psi_{n_{k-1} l_{k-1} m_{k-1}}, \ldots, \psi_{n_{1}, l_{1}, m_{1}}$ are different from the state $\psi_{n, l, m}$. Using the identities (33), it may now be checked that the expression in Eq. (35) is equivalent to the following:

$$
(e \mathcal{E})^{k+1}\left\langle\psi_{n l m}|x| \psi_{n_{k} l_{k} m_{k}}\right\rangle\left\langle\psi_{n_{k} l_{k} m_{k}}|x| \psi_{n_{k-1} l_{k-1} m_{k-1}}\right\rangle \ldots\left\langle\psi_{n_{2} l_{2} m_{2}}|x| \psi_{n_{1} l_{1} m_{1}}\right\rangle\left\langle\psi_{n_{1} l_{1} m_{1}}|x| \psi_{n l m}\right\rangle
$$

Further, for the application of the degenerate perturbation theory for $n>1$, the elements of the $n^{2} \times n^{2}$ matrix $M$ determining the secular equation is of the form:

$$
[M]_{i j}=a_{i j} e^{\gamma_{i}-\gamma_{j}}, \quad a_{i j}=a_{j i} \in R, \quad \gamma_{i} \in R .
$$


Any matrix of this type can be shown to be pseudo-symmetric, i.e. $M$ is related to its transpose $M^{T}$ though a similarity transformation, $M^{T}=\eta M \eta^{-1}$, with the similar matrix $\eta$ being given by, $[\eta]_{i j}=e^{-2 \gamma_{i}} \delta_{i j}$. Consequently, $M$ can be transformed to a symmetric matrix $\mathcal{M}$ as $\mathcal{M}=\rho M \rho^{-1}$ with $\rho:=\sqrt{M}$ and $[\mathcal{M}]_{i j}=a_{i j}$. If the degenerate perturbation theory is applied to $h$ with $e \mathcal{E} x$ as the perturbation, the matrix determining the secular equation is precisely of the form $\mathcal{M}$. Thus, the perturbative analysis of $H$ and $h$ gives identical results at each order of the perturbation.

A comment is in order before the end of this section. The Hamiltonian $H_{0}$ is also hermitian in the Hilbert space $\mathcal{H}_{\eta_{+}}$. A complete set of orthonormal states of the Hamiltonian $H_{0}$ with the energy $E_{n}$ may be constructed in the Hilbert space $\mathcal{H}_{\eta_{+}}$as, $\phi_{n l m}=(U \rho)^{-1} \psi_{n l m}$. The perturbing term $H^{\prime}=e \mathcal{E} X$ is also hermitian in $\mathcal{H}_{\eta_{+}}$and the states $\phi_{n l m}$ can be used to calculate perturbative corrections at different orders to the energy $E_{n}$. The corrections to the energy eigenvalues may be obtained by replacing the standard inner product $\langle.,$.$\rangle with$ the modified inner product $\langle\langle., .\rangle\rangle_{\eta_{+}}$. The identity,

$$
\left\langle\left\langle\phi_{n l m}|X| \phi_{n^{\prime} l^{\prime} m^{\prime}}\right\rangle\right\rangle_{\eta_{+}}=\left\langle\psi_{n l m}|x| \psi_{n^{\prime} l^{\prime} m^{\prime}}\right\rangle
$$

is useful in establishing one to one correspondence between the perturbative corrections of

$H$ and $h$ at each order. For example, the expansion of the ground-state energy $\tilde{E}_{1}$ of $H$ up to the second order is obtained as,

$$
\begin{aligned}
\tilde{E}_{1} & =E_{1}+e \mathcal{E}\left\langle\left\langle\phi_{100}|X| \phi_{100}\right\rangle\right\rangle_{\eta_{+}}+(e \mathcal{E})^{2} \sum_{n(\neq 1), l, m} \frac{\left|\left\langle\left\langle\phi_{100}|X| \phi_{n l m}\right\rangle\right\rangle_{\eta_{+}}\right|^{2}}{E_{1}-E_{n}} \\
& =E_{1}+e \mathcal{E}\left\langle\psi_{100}|x| \psi_{100}\right\rangle+(e \mathcal{E})^{2} \sum_{n(\neq 1), l, m} \frac{\left|\left\langle\psi_{100}|x| \psi_{n l m}\right\rangle\right|^{2}}{E_{1}-E_{n}}
\end{aligned}
$$

The results of perturbative analysis of $H$ either in $\mathcal{H}_{D}$ or $\mathcal{H}_{\eta_{+}}$would give identical results at each order of perturbation.

\section{Zeeman effect}

A hermitian Hamiltonian in $\mathcal{H}_{\eta_{+}}$describing Zeeman effect may be constructed as follows:

$$
H=\frac{P^{2}}{2 m}-\frac{e^{2}}{R}+\frac{1}{2 m^{2} R} \frac{d V(R)}{d R} \vec{L} \cdot \vec{T}+\frac{e}{2 m} \vec{B} \cdot(\vec{L}+2 \vec{T})+\frac{e^{2}}{8 m}(\vec{B} \times \vec{R})^{2},
$$


where $\vec{B}$ is external uniform magnetic field and $V(R)$ is a real function of its argument which can be chosen to be Coulomb potential. Unlike the case of Stark effect where the external electric field is complex, the magnetic field $\vec{B}$ describing Zeeman effect is real both in $\mathcal{H}_{D}$ as well as in $\mathcal{H}_{\eta_{+}}$. It should be mentioned here that the vector potential producing the real magnetic field is not necessarily real in $\mathcal{H}_{D}$ for which the relevant position operators are $(x, y, z)$. For example, the vector potential $\vec{A}$ with components $A_{x}=\frac{B}{2}(i x \sinh w-y \cosh w), A_{y}=\frac{B}{2}(x \cosh w+i y \sinh w)$ and $A_{z}=0$ produces real magnetic field along the $z$-direction. Both $A_{x}$ and $A_{y}$ have a real part and an imaginary part. The study of quantum mechanical systems with imaginary gauge potential has relevance in understanding different kinds of phase transitions[31]. Thus, the consideration of complex gauge potential is physically well motivated.

The Hamiltonian is non-hermitian in $\mathcal{H}_{D}$, as can be seen by rewriting it in terms of the variables $x, y, z, \mathcal{L}_{x, y, z}, \mathcal{S}_{x, y, z}$. The Hamiltonian is hermitian in both $\mathcal{H}_{D}$ as well as in $\mathcal{H}_{\eta_{+}}$ in the following two limits: (i) $\gamma=\delta=0$ and (ii) $\gamma=\delta, \xi=\chi, \vec{B}=|\vec{B}| \hat{k}$, where $\hat{k}$ is a unit vector along the $z$-direction. The second limit is interesting in the following sense. The kinetic energy and the Coulomb potential terms are hermitian both in $\mathcal{H}_{D}$ as well as in $\mathcal{H}_{\eta_{+}}$without any restriction on the parameters. With the choice of $\gamma=\delta, \xi=\chi$, the spin-orbit interaction term of the Hamiltonian is hermitian in $\mathcal{H}_{D}$ as well as in $\mathcal{H}_{\eta_{+}}$. The origin of non-hermiticity of the last two terms in $\mathcal{H}_{D}$ is physically well motivated through the introduction of imaginary gauge potential. These two terms also become hermitian in $\mathcal{H}_{D}$, if the magnetic field is taken along the $z$-direction. Thus, the direction of the external magnetic field can be varied to switch over from hermitian to non-hermitian description of $H$ in $\mathcal{H}_{D}$

The equivalent hermitian Hamiltonian $h:=(U \rho) H(U \rho)^{-1}$ in $\mathcal{H}_{D}$ has the following form:

$$
h=\frac{p^{2}}{2 m}-\frac{e^{2}}{r}+\frac{1}{2 m^{2} r} \frac{d V(r)}{d r} \overrightarrow{\mathcal{L}} \cdot \overrightarrow{\mathcal{S}}+\frac{e}{2 m} \vec{B} \cdot(\overrightarrow{\mathcal{L}}+2 \overrightarrow{\mathcal{S}})+\frac{e^{2}}{8 m}(\vec{B} \times \vec{r})^{2} .
$$

Both $H$ and $h$ are isospectral and electromagnetic transition rate for given two states. However, the eigenfunctions are different from each other. The study on the eigenvalue problem of $h$ is included in any standard book on quantum mechanics and thus, no discussion 
in this regard is given in this paper. Further, a direct perturbative analysis of $H$ either in the Hilbert space $\mathcal{H}_{D}$ or $\mathcal{H}_{\eta_{+}}$may be carried out following the discussions in the previous section on Stark effect.

An experimental realization or verification of the predictions emanating from the study of pseudo-hermitian $/ \mathcal{P} \mathcal{T}$-symmetric quantum mechanics is desirable. In this regard, the examples considered in this section may offer promising scenarios. If non-hermitian interactions of the form described in this paper can be produced in the laboratory with $\gamma$ being one of the externally controllable parameters, transition rate between two allowed levels may be studied for $\gamma=0$ and $\gamma \neq 0$. It may be recalled here that in the Hilbert space $\mathcal{H}_{D}, \gamma=0$ and $\gamma \neq 0$ correspond to hermitian and non-hermitian Hamiltonian, respectively. According to the prediction of this paper, the transition rate between any two allowed levels would be independent of $\gamma$, if nature realizes pseudo-hermitian $/ \mathcal{P} \mathcal{T}$-symmetric quantum systems.

\section{EXAMPLES: MANY BODY SYSTEM}

In this section, examples from many body quantum systems are considered. General quadratic form of $N$ bosons(fermions) with non-hermitian interactions, symmetric and asymmetric XXZ spin chain Hamiltonian in an external uniform, complex magnetic field are considered in this section. A non-hermitian version of Haldane-Shastry spin-chain and Lipkin-Meshkov-Glick model is also discussed.

\section{A. Hamiltonian: General Quadratic Form of Boson Operators}

General quadratic form of $N$ boson operators satisfying the commutation relations,

$$
\left[a_{i}, a_{j}^{\dagger}\right]=\delta_{i j}, \quad\left[a_{i}, a_{j}\right]=0=\left[a_{i}^{\dagger}, a_{j}^{\dagger}\right], \quad i, j=1,2, \ldots, N
$$

appear in many diverse branches of physics. The operator $a_{i}^{\dagger}$ is the adjoint of $a_{i}$ in the Hilbert space $\mathcal{H}_{D}$ and $a_{i}\left(a_{i}^{\dagger}\right)$ may be identified as the annihilation(creation) operator. A non- 
hermitian general quadratic form involving these operators may be constructed as follows,

$$
\begin{aligned}
& H=\frac{1}{2} \sum_{i, j=1}^{N}\left[\alpha_{i j}\left(e^{w_{i}-w_{j}} a_{i}^{\dagger} a_{j}+e^{-\left(w_{i}-w_{j}\right)} a_{j}^{\dagger} a_{i}\right)+\beta_{i j}\left(e^{-\left(w_{i}+w_{j}\right)} a_{i} a_{j}+e^{w_{i}+w_{j}} a_{i}^{\dagger} a_{j}^{\dagger}\right)\right] \\
& w_{i} \equiv \gamma_{i}+i \xi_{i}, \quad\left\{\gamma_{i}, \xi_{i} \alpha_{i j}, \beta_{i j}\right\} \in R, \alpha_{i j}=\alpha_{j i}, \beta_{i j}=\beta_{j i} .
\end{aligned}
$$

In a coordinate space realization of the algebra (42), $H$ corresponds to a quantum system of $N$ simple harmonic oscillators interacting with each other through non-hermitian interaction in one dimension. Alternatively, the same Hamiltonian $H$ can be identified as that of a $N$ dimensional oscillator with non-hermitian interaction. The non-hermitian interactions in Eq. (43) may be interpreted as arising due to imaginary gauge potential. It may be noted that such imaginary gauge potentials are also relevant in the context of metal-insulator transitions or depinning of flux-lines from extended defects in type-II superconductors[31]. In fact, with nearest-neighbor interaction only and $\beta_{i j}=0 \forall i, j, H$ resembles randomhopping model of Ref. [31]. For $N=1, H$ is known as Swanson Hamiltonian[6] and has been studied extensively in the literature in the context of $\mathcal{P} \mathcal{T}$ symmetric and pseudohermitian quantum system. It is worth mentioning here that a non-hermitian $\mathcal{P} \mathcal{T}$-symmetric two-mode Bose-Hubbard system has been studied in Ref. [22]. The Hamiltonian in Ref. [22] is different from the Hamiltonian in Eq. (43).

The claim of this paper is that the non-hermitian $H$ in Eq. (43) admits entirely real spectra with unitary time evolution for arbitrary $N$ and within a fixed region in the parameterspace. To substantiate this claim, the metric operator $\eta_{+}$and the similar operator $\rho:=\sqrt{\eta_{+}}$ may be introduced as,

$$
\eta_{+}:=\prod_{i=1}^{N} e^{-2 \gamma_{i} a_{i}^{\dagger} a_{i}}, \quad \rho:=\prod_{i=1}^{N} e^{-\gamma_{i} a_{i}^{\dagger} a_{i}} .
$$

A set of operators $A_{i}$ and their adjoint $A_{i}^{\dagger}$ in the Hilbert space of $\mathcal{H}_{\eta_{+}}$is introduced as follows:

$$
A_{i}:=\rho^{-1} a_{i} \rho=e^{-\gamma_{i}} a_{i}, \quad A_{i}^{\dagger}:=\rho^{-1} a_{i}^{\dagger} \rho=e^{\gamma_{i}} a_{i}^{\dagger},
$$

which satisfy the same algebra given by Eq. (42). A general eigen state of the total boson number operator in the Hilbert space $\mathcal{H}_{D}$ may be introduced as $\left|n_{1}, \ldots, n_{i}, \ldots, n_{N}\right\rangle_{\mathcal{H}_{D}}$ with 
the following relations:

$$
\begin{aligned}
& a_{i}\left|n_{1}, \ldots, n_{i}, \ldots, n_{N}\right\rangle_{\mathcal{H}_{D}}=\sqrt{n_{i}}\left|n_{1}, \ldots, n_{i}-1, \ldots, n_{N}\right\rangle_{\mathcal{H}_{D}} \\
& a_{i}^{\dagger}\left|n_{1}, \ldots, n_{i}, \ldots, n_{N}\right\rangle_{\mathcal{H}_{D}}=\sqrt{n_{i}+1}\left|n_{1}, \ldots, n_{i}+1, \ldots, n_{N}\right\rangle_{\mathcal{H}_{D}} .
\end{aligned}
$$

The corresponding state in the the Hilbert space $\mathcal{H}_{\eta_{+}}$is determined as,

$$
\left|n_{1}, \ldots, n_{i}, \ldots, n_{N}\right\rangle_{\mathcal{H}_{\eta_{+}}}=\prod_{k=1}^{N} e^{\gamma_{k} n_{k}}\left|n_{1}, \ldots, n_{i}, \ldots, n_{N}\right\rangle_{\mathcal{H}_{D}}
$$

with the action of $A_{i}\left(A_{i}^{\dagger}\right)$ on $\left|n_{1}, \ldots, n_{i}, \ldots, n_{N}\right\rangle_{\mathcal{H}_{\eta_{+}}}$given by the following relations:

$$
\begin{aligned}
& A_{i}\left|n_{1}, \ldots, n_{i}, \ldots, n_{N}\right\rangle_{\mathcal{H}_{\eta_{+}}}=\sqrt{n_{i}}\left|n_{1}, \ldots, n_{i}-1, \ldots, n_{N}\right\rangle_{\mathcal{H}_{\eta_{+}}} \\
& A_{i}^{\dagger}\left|n_{1}, \ldots, n_{i}, \ldots, n_{N}\right\rangle_{\mathcal{H}_{\eta_{+}}}=\sqrt{n_{i}+1}\left|n_{1}, \ldots, n_{i}+1, \ldots, n_{N}\right\rangle_{\mathcal{H}_{\eta_{+}}} .
\end{aligned}
$$

The states $\left|n_{1}, \ldots, n_{i}, \ldots, n_{N}\right\rangle_{\mathcal{H}_{\eta_{+}}}$form a complete set of orthonormal states in $\mathcal{H}_{\eta_{+}}$, while $\left|n_{1}, \ldots, n_{i+1}, \ldots, n_{N}\right\rangle_{\mathcal{H}_{D}}$ form a complete set of orthonormal states in $\mathcal{H}_{D}$.

The Hamiltonian $H$ is hermitian in $\mathcal{H}_{\eta_{+}}$and this can be checked easily by rewriting it as,

$$
H=\frac{1}{2} \sum_{i, j=1}^{N}\left[\alpha_{i j}\left(e^{i\left(\xi_{i}-\xi_{j}\right)} A_{i}^{\dagger} A_{j}+e^{-i\left(\xi_{i}-\xi_{j}\right)} A_{j}^{\dagger} A_{i}\right)+\beta_{i j}\left(e^{-i\left(\xi_{i}+\xi_{j}\right)} A_{i} A_{j}+e^{i\left(\xi_{i}+\xi_{j}\right)} A_{i}^{\dagger} A_{j}^{\dagger}\right)\right] .
$$

The Hamiltonian $H$ can be mapped to a Hamiltonian $h$ that is hermitian in $\mathcal{H}_{D}$,

$$
\begin{aligned}
h & =\rho H \rho^{-1} \\
& =\frac{1}{2} \sum_{i, j=1}^{N}\left[\alpha_{i j}\left(e^{i\left(\xi_{i}-\xi_{j}\right)} a_{i}^{\dagger} a_{j}+e^{-i\left(\xi_{i}-\xi_{j}\right)} a_{j}^{\dagger} a_{i}\right)+\beta_{i j}\left(e^{-i\left(\xi_{i}+\xi_{j}\right)} a_{i} a_{j}+e^{i\left(\xi_{i}+\xi_{j}\right)} a_{i}^{\dagger} a_{j}^{\dagger}\right)\right],
\end{aligned}
$$

thereby showing the quasi-hermiticity of $H$. A further unitary transformation removes the phase-factors from $h$. In particular,

$$
\begin{aligned}
U & :=\prod_{i=1}^{N} e^{-i \xi_{i} a_{i}^{\dagger} a_{i}} \\
\tilde{h} & =U h U^{-1}=\frac{1}{2} \sum_{i, j=1}^{N}\left[\alpha_{i j}\left(a_{i}^{\dagger} a_{j}+a_{j}^{\dagger} a_{i}\right)+\beta_{i j}\left(a_{i} a_{j}+a_{i}^{\dagger} a_{j}^{\dagger}\right)\right] .
\end{aligned}
$$

A general prescription to diagonalize (51) has been given in Ref. [32]. The basic steps involve the identification of the following $2 N \times 2 N$ matrices,

$$
D=\left(\begin{array}{cc}
\hat{\alpha} & \hat{\beta} \\
\hat{\beta} & \hat{\alpha}
\end{array}\right), \quad \hat{I}=\left(\begin{array}{cc}
I & 0 \\
0 & -I
\end{array}\right), \quad Q:=\hat{I} D=\left(\begin{array}{cc}
\hat{\alpha} & -\hat{\beta} \\
\hat{\beta} & -\hat{\alpha}
\end{array}\right) \text {, }
$$


where $I$ is $N \times N$ Identity matrix, $\hat{\alpha}$ and $\hat{\beta}$ are $N \times N$ matrices with the elements $[\hat{\alpha}]_{i j}=\alpha_{i j}$ and $[\hat{\beta}]_{i j}=\beta_{i j}$. It can be shown that the eigenvalues of the matrix $Q$ are of the form $\Omega \in\left\{\Omega_{1}, \Omega_{2}, \ldots, \Omega_{N},-\Omega_{1},-\Omega_{2}, \ldots,-\Omega_{N}\right\}$. Further, if $\hat{u}_{i}$ is the eigen-vector corresponding to the eigenvalue $\Omega_{i}$ of $Q$, then, $-\Omega_{i}$ is an another eigen value of $Q$ with the eigen-vector $\hat{J} \hat{u}_{i}$, where $\hat{J}$ is an anti-linear, idempotent operator that commutes with $D$ and anti-commutes with $\hat{I}[32]$. The energy eigenvalues of $\tilde{h}$ are,

$$
E_{\left\{n_{i}\right\}}=\sum_{i=1}^{N}\left(n_{i}+\frac{1}{2}\right) \Omega_{i}, \quad \Omega_{i}>0 \forall i .
$$

The stability criteria requires a positive-definite $\Omega_{i}$ and consequently, these results are valid only in those regions in the parameter-space for which $D$ is strictly positive[32].

A comment is in order before the end of this section. Schwinger's oscillator model of angular momentum can be realized in terms of $A_{1}, A_{2}$ and their adjoint in $\mathcal{H}_{\eta_{+}}$. The following angular momentum operators satisfying $S U(2)$ algebra may be defined,

$$
\begin{aligned}
\hat{J}_{+} & :=A_{1}^{\dagger} A_{2}=e^{\gamma_{1}-\gamma_{2}} a_{1}^{\dagger} a_{2}, \\
\hat{J}_{-} & :=A_{2}^{\dagger} A_{1}=e^{-\left(\gamma_{1}-\gamma_{2}\right)} a_{2}^{\dagger} a_{1}, \\
\hat{J}_{z} & :=\frac{1}{2}\left(A_{1}^{\dagger} A_{1}-A_{2}^{\dagger} A_{2}\right)=\frac{1}{2}\left(a_{1}^{\dagger} a_{1}-a_{2}^{\dagger} a_{2}\right),
\end{aligned}
$$

where $\hat{J}_{+}$is the adjoint of $\hat{J}_{-}$in $\mathcal{H}_{\eta_{+}}$. The operator $\hat{J}_{z}$ is hermitian in $\mathcal{H}_{D}$ as well as in $\mathcal{H}_{\eta_{+}}$. The usual physical interpretation of the Schwinger's oscillator model of angular momentum is equally applicable to the generators $\hat{J}_{ \pm}, \hat{J}_{z}$ with the help of Eqs. (466), (477) and (48). Suitable combinations of these operators would result in pseudo-hermitian Hamiltonian with the metric $\eta_{+}$. One such example is the non-hermitian deformation of the Lipkin-MeshkovGlick(LMG) model[24],

$$
H_{L M G}=\omega_{0} \hat{J}_{z}+\omega\left(\hat{J}_{-}^{2}+\hat{J}_{+}^{2}\right),
$$

where $\omega_{0}$ and $\omega$ are real parameters. In the hermitian limit, $\gamma_{1}=\gamma_{2}=0$, the standard LMG model is reproduced which has been studied extensively in the literature[33]. For $\gamma_{1} \neq 0 \neq \gamma_{2} H_{L M G}$ is isospectral with the standard LMG model. 


\section{B. Hamiltonian: General Quadratic Form of Fermion Operators}

A set of canonical Fermi operators satisfying the anti-commutation relations,

$$
\left\{c_{i}, c_{j}^{\dagger}\right\}=2 \delta_{i j}, \quad\left\{c_{i}, c_{j}\right\}=0=\left\{c_{i}^{\dagger}, c_{j}^{\dagger}\right\}, \quad i, j=1,2, \ldots N
$$

and a non-hermitian Hamiltonian in terms of these operators may be introduced in $\mathcal{H}_{D}$ as follows:

$$
\begin{aligned}
H & =\sum_{i, j=1}^{N} A_{i j} c_{i}^{\dagger} c_{j} e^{w_{i}-w_{j}}+\frac{1}{2} \sum_{i, j=1}^{N} B_{i j}\left(c_{i}^{\dagger} c_{j}^{\dagger} e^{\left(w_{i}+w_{j}\right)}+c_{i} c_{j} e^{-\left(w_{i}+w_{j}\right)}\right), \\
A_{i j} & =A_{j i} \in R, \quad B_{i j}=-B_{j i} \in R .
\end{aligned}
$$

The complex parameters $w_{i}$ 's are defined in Eq. (43). The Hamiltonian $H$ is hermitian in $\mathcal{H}_{\eta_{+}}$with the metric $\eta_{+}$defined as,

$$
\eta_{+}:=\prod_{i=1}^{N} e^{-2 \gamma_{i} c_{i}^{\dagger} c_{i}}
$$

The Hamiltonian can be mapped to a hermitian Hamiltonian $h$ in $\mathcal{H}_{D}$ by using the similar operator $\rho:=\prod_{i=1}^{N} e^{-\gamma_{i} c_{i}^{\dagger} c_{i}}$ and a unitary operator $U:=\prod_{i=1}^{N} e^{-i \xi_{i} c_{i}^{\dagger} c_{i}}$ as,

$$
h:=(U \rho) H(U \rho)^{-1}=\sum_{i, j=1}^{N} A_{i j} c_{i}^{\dagger} c_{j}+\frac{1}{2} \sum_{i, j=1}^{N} B_{i j}\left(c_{i}^{\dagger} c_{j}^{\dagger}+c_{i} c_{j}\right) .
$$

The Hamiltonian $h$ is exactly solvable and the diagonalization procedure is described in detail in Ref. [34]. For nearest-neighbor interaction $H(h)$ can be mapped to a solvable nonhermitian(hermitian) $X Y$ spin chain in $\mathcal{H}_{D}$ by using the Jordan-Wigner transformation[34].

The fermionic annihilation operators $C_{i}$ and their adjoint $C_{i}^{\dagger}$ in $\mathcal{H}_{\eta_{+}}$may be defined in terms of $c_{i}, c_{i}^{\dagger}$ as,

$$
C_{i}:=e^{-\gamma_{i}} c_{i}, \quad C_{i}^{\dagger}:=e^{\gamma_{i}} c_{i}^{\dagger},
$$

which satisfy the basic canonical anti-commutation relations (156). A general eigen state of the total fermion number operator in the Hilbert space $\mathcal{H}_{D},\left|f_{1}, \ldots, f_{i}, \ldots, f_{N}\right\rangle_{\mathcal{H}_{D}}$ is related to the corresponding state $\left|f_{1}, \ldots, f_{i}, \ldots, f_{N}\right\rangle_{\mathcal{H}_{\eta_{+}}}$in the Hilbert space $\mathcal{H}_{\eta_{+}}$through the following relation:

$$
\left|f_{1}, \ldots, f_{i}, \ldots, f_{N}\right\rangle_{\mathcal{H}_{\eta_{+}}}=\prod_{k=1}^{N} e^{\gamma_{k} f_{k}}\left|f_{1}, \ldots, f_{i}, \ldots, f_{N}\right\rangle_{\mathcal{H}_{D}}, \quad f_{i}=0,1 \forall i .
$$


The $2^{N}$ states $\left|f_{1}, \ldots, f_{i}, \ldots, f_{N}\right\rangle_{\mathcal{H}_{\eta_{+}}}$form a complete set of orthonormal states in $\mathcal{H}_{\eta_{+}}$, while $\left|f_{1}, \ldots, f_{i}, \ldots, f_{N}\right\rangle_{\mathcal{H}_{D}}$ constitute a complete set of orthonormal states in $\mathcal{H}_{D}$. The action of $C_{i}\left(C_{i}^{\dagger}\right)$ on $\left|f_{1}, \ldots, f_{i}, \ldots, f_{N}\right\rangle_{\mathcal{H}_{\eta_{+}}}$is identical to that of $c_{i}\left(c_{i}^{\dagger}\right)$ on $\left|f_{1}, \ldots, f_{i}, \ldots, f_{N}\right\rangle_{\mathcal{H}_{D}}$. In particular,

$$
\begin{aligned}
C_{i}\left|f_{1}, \ldots, f_{i}, \ldots, f_{N}\right\rangle_{\mathcal{H}_{\eta_{+}}} & =0, \text { if } f_{i}=0 \\
& =\left|f_{1}, \ldots, 0, \ldots, f_{N}\right\rangle_{\mathcal{H}_{\eta_{+}}}, \text {if } f_{i}=1 ; \\
C_{i}^{\dagger}\left|f_{1}, \ldots, f_{i}, \ldots, f_{N}\right\rangle_{\mathcal{H}_{\eta_{+}}} & =0, \text { if } f_{i}=1 \\
& =\left|f_{1}, \ldots, 1, \ldots, f_{N}\right\rangle_{\mathcal{H}_{\eta_{+}}}, \text {if } f_{i}=0 .
\end{aligned}
$$

Suitable combinations of the operators $C_{i}$ and $C_{i}^{\dagger}$ would give rise to a very large number of pseudo-hermitian quantum systems that go beyond general quadratic form of fermionic oscillators. Further, the definitions of $C_{i}, C_{i}^{\dagger}$ could be generalized easily to accommodate a pseudo-hermitian description of Hubbard model, t-j model etc. As in the case of bosonic oscillators, the $S U(2)$ generators can be realized in terms of pseudo-hermitian fermion operators.

\section{XXZ Spin-chain}

The study of non-hermitian spin chains has a long history. It is a well known fact that non-hermitian quantum spin chains correspond to two-dimensional classical systems with positive Boltzmann weights. The non-hermitian XY and XXZ spin chain Hamiltonians with Dzyaloshinsky-Moriya interaction commute with the transfer matrix of the six-vertex model in the presence of an electric field[35] and, the integrable chiral Potts model in the most general case leads to a non-hermitian quantum Hamiltonian[36, 37]. Non-hermitian asymmetric $X X Z$ spin chains related to diffusion models have been studied extensively in non-equilibrium statistical mechanics[38]. Further, a non-hermitian quantum Ising spin chain in one dimension [39] is known to be related to the celebrated Yang-Lee model[40] that aptly describes ordinary second order phase transitions. The non-hermiticity of the spin chain arises due to the inclusion of an external complex magnetic field and an analysis based 
on minimal conformal field theory is available[41]. Within the context of $\mathcal{P} \mathcal{T}$-symmetric theory, non-hermitian spin chains have been studied in Refs. [8, 9].

The pseudo-hermitian spin operators $T_{x, y, z}$ and the metric operator $\zeta_{+}$, as given in Eqs. (16) and (17), may be generalized appropriately to introduce pseudo-hermitian XXZ spinchain Hamiltonian. One such simple generalization is to consider the spin operators $T_{i}^{x, y, z}$,

$$
\begin{aligned}
T_{i}^{x} & :=\cosh _{i} \mathcal{S}_{i}^{x}+i \sinh w_{i} \mathcal{S}_{i}^{y} \\
T_{i}^{y} & :=-i \sinh w_{i} \mathcal{S}_{i}^{x}+\cosh _{i} \mathcal{S}_{i}^{y} \\
T_{i}^{z} & :=\mathcal{S}_{i}^{z}
\end{aligned}
$$

which are hermitian in the Hilbert space $\mathcal{H}_{\zeta_{+}}$with the positive-definite metric $\zeta_{+}$defined as,

$$
\zeta_{+}:=\prod_{i=1}^{N} e^{-2 \gamma_{i} T_{i}^{z}}
$$

The operators $\mathcal{S}_{i}^{x, y, z}$ are hermitian in the Hilbert space $\mathcal{H}_{D}$ with the standard inner product. An asymmetric XXZ spin-chain in an external complex magnetic field may now be constructed that is manifestly non-hermitian in $\mathcal{H}_{D}$,

$$
\begin{aligned}
H_{A} & =\sum_{i=1}^{N-1}\left[\Gamma\left(e^{w_{i}-w_{i+1}} \mathcal{S}_{i}^{+} \mathcal{S}_{i+1}^{-}+e^{-\left(w_{i}-w_{i+1}\right)} \mathcal{S}_{i}^{-} \mathcal{S}_{i+1}^{+}\right)+\Delta \mathcal{S}_{i}^{z} \mathcal{S}_{i+1}^{z}\right. \\
& \left.+\left(A_{i} \cosh _{i}-i B_{i} \sinh w_{i}\right) \mathcal{S}_{i}^{x}+\left(B_{i} \cosh w_{i}+i A_{i} \sinh w_{i}\right) \mathcal{S}_{i}^{y}+C_{i} \mathcal{S}_{i}^{z}\right]
\end{aligned}
$$

where $\mathcal{S}_{i}^{ \pm}:=\mathcal{S}_{i}^{x} \pm i \mathcal{S}_{i}^{y},\left\{\Gamma, \Delta, A_{i}, B_{i}, C_{i}\right\} \in R$ and $w_{i}$ are as defined in Eq. (43). The non-hermitian interaction in $H_{A}$ may be interpreted as arising due to imaginary vector potential as in the case of Bose system described before. In fact, with a hard-core boson representation, $H_{A}$ can be mapped to a nearest-neighbor version of $H$ in Eq. (43).

The Hamiltonian $H_{A}$ can be mapped to a hermitian Hamiltonian in $\mathcal{H}_{D}$,

$$
\begin{aligned}
h & :=U\left(\zeta_{+}^{\frac{1}{2}} H_{A} \zeta_{+}^{-\frac{1}{2}}\right) U^{-1} \\
& =\sum_{i=1}^{N-1}\left[\Gamma\left(\mathcal{S}_{i}^{x} \mathcal{S}_{i+1}^{x}+\mathcal{S}_{i}^{y} \mathcal{S}_{i+1}^{y}\right)+\Delta \mathcal{S}_{i}^{z} \mathcal{S}_{i+1}^{z}+A_{i} \mathcal{S}_{i}^{x}+B_{i} \mathcal{S}_{i}^{y}+C_{i} \mathcal{S}_{i}^{z}\right], U:=\prod_{i=1}^{N} e^{-i \chi_{i} \mathcal{S}_{i}^{z}(66)}
\end{aligned}
$$

implying that both $H_{A}$ and $h$ have entirely real spectra. The asymmetric $X X Z$ spin-chain Hamiltonian $H_{A}$ is hermitian in $\mathcal{H}_{\eta_{+}}$and this may be checked easily by rewriting $H_{A}$ as,

$$
H_{A}=\sum_{i=1}^{N-1}\left[\Gamma\left(T_{i}^{+} T_{i+1}^{-}+T_{i}^{-} T_{i+1}^{+}\right)+\Delta T_{i}^{z} T_{i+1}^{z}+A_{i} T_{i}^{x}+B_{i} T_{i}^{y}+C_{i} T_{i}^{z}\right],
$$


where $T_{i}^{ \pm}:=T_{i}^{x} \pm i T_{i}^{y}$. Thus, the time-evolution of $H_{A}$ is unitary in $\mathcal{H}_{\eta_{+}}$.

A few comments are in order at this point.

(i) Several variants of the asymmetric XXZ Hamiltonian (65) have been studied in the literature[38] in the context of two species reaction-diffusion processes and Kardar-ParisiZhang-type growth phenomenon. A typical choice for $w_{k}$ in these models is,

$$
\gamma_{k}=\gamma-(k-1) \phi, \quad \xi_{k}=\xi \forall k, \quad\{\gamma, \xi, \phi\} \in R
$$

leading to a site-independent global phase factor $e^{ \pm \phi}$ in lieu of $e^{ \pm\left(w_{i}-w_{i+1}\right)}$. The transformation that maps non-hermitian asymmetric $X X Z$ Hamiltonian to a hermitian Hamiltonian is also known in the literature[38]. This transformation is generally used to show the reality of the entire spectra. However, with the standard inner product in the Hilbert space $\mathcal{H}_{D}$, negative norm states exist. Consequently, in spite of having an entirely real spectra, the time-evolution of $H_{A}$ in $\mathcal{H}_{D}$ is not unitary and dissipative processes thus can be stimulated. The pseudo-hermiticity of $H_{A}$ has not been noted previously. The time-evolution of $H_{A}$ in $\mathcal{H}_{\eta_{+}}$is unitary. Thus, with the discovery of the pseudo-hermiticity of $H_{A}$, it may be used to describe unitary time evolution in $\mathcal{H}_{\eta_{+}}$. At a purely formal level, it might seem to be a matter of choice to describe either unitary or non-unitary time-evolution by fixing an appropriate metric in the Hilbert space. However, an experimental realization of any one of these systems may give a definite answer on whether nature realizes pseudo-hermitian quantum systems or not.

(ii) The symmetric $X X Z$ spin-chain Hamiltonian in an external complex magnetic field may be constructed by choosing $w_{i} \equiv w \equiv \gamma+i \chi \forall i,\{\gamma, \chi\} \in R$ in Eq. (65),

$$
\begin{aligned}
H_{S} & =\sum_{i=1}^{N-1}\left[\Gamma\left(\mathcal{S}_{i}^{x} \mathcal{S}_{i+1}^{x}+\mathcal{S}_{i}^{y} \mathcal{S}_{i+1}^{y}\right)+\Delta \mathcal{S}_{i}^{z} \mathcal{S}_{i+1}^{z}+\left(A_{i} \cosh w-i B_{i} \sinh w\right) \mathcal{S}_{i}^{x}\right. \\
& \left.+\left(B_{i} \cosh w+i A_{i} \sinh w\right) \mathcal{S}_{i}^{y}+C_{i} \mathcal{S}_{i}^{z}\right]
\end{aligned}
$$

which is non-hermitian in $\mathcal{H}_{D}$, but, is hermitian in $\mathcal{H}_{\zeta_{+}}$. The equivalent hermitian Hamiltonian $h:=U\left(\zeta_{+}^{\frac{1}{2}} H_{S} \zeta_{+}^{-\frac{1}{2}}\right) U^{-1}$ in $\mathcal{H}_{D}$ to $H_{S}$ is still given by Eq. (66).

The Hamiltonian $h$ has several integrable limits. Consequently, $H_{A}$ and $H_{S}$ are also integrable in these limits with entirely real spectra and unitary time-evolution. For example, 
$h$ reduces to a transverse-field Ising model for $\Gamma=B_{i}=C_{i}=0, A_{i}=A \forall i$ and both $h$ and $H_{S}$ have been studied in some detail[8] for this limiting case. For $\Delta=0, A_{i}=0, B_{i}=0 \forall i$, $h$ reduces to an XX model in a transverse magnetic field and is exactly solvable[34, 42]. Although $H_{S}$ is hermitian in $\mathcal{H}_{D}$ for this choice of the parameters, $H_{A}$ is non-hermitian. Thus, the non-hermitian $H_{A}$ is exactly solvable and has an equivalent description in terms of a hermitian XX model in an external magnetic field. For the following choice of the parameters,

$$
\Gamma=1, \Delta=\cosh q, C_{1}=-C_{N}=-\sinh q, A_{i}=B_{i}=0 \forall i ; C_{i}=0, i=2,3, \ldots, N-1,
$$

$h-\Delta$ reduces to an $S U_{q}(2)$ invariant [43] integrable[44] spin-chain Hamiltonian. The XXZ spin-chain with $S l_{2}$ loop symmetry[45] may also be obtained as a limiting case. The corresponding non-hermitian Hamiltonian $H_{A}$ is also integrable and allows an unitary description.

(iii) Only the spin-chains with nearest-neighbor interactions are presented in this paper. A large number of pseudo-hermitian spin-chains with no restriction on the type of interactions (i.e. nearest-neighbor, next-nearest neighbor etc. ) may be constructed by the use of the operators $T_{i}^{x, y, z}$. For example, a non-hermitian version of the celebrated Haldane-Shastry spin-chain [23] may be constructed as follows:

$$
H= \pm \sum_{i<j} \frac{\vec{T}_{i} \cdot \vec{T}_{j}}{2 \sin ^{2} \frac{\pi}{N}(i-j)}
$$

where $H$ is hermitian in $\mathcal{H}_{\eta_{+}}$and non-hermitian in $\mathcal{H}_{D}$. The equivalent hermitian Hamiltonian in $\mathcal{H}_{D}$ may be obtained as,

$$
h:=(U \rho) H(U \rho)^{-1}= \pm \sum_{i<j} \frac{\overrightarrow{\mathcal{S}}_{i} \cdot \overrightarrow{\mathcal{S}}_{j}}{2 \sin ^{2} \frac{\pi}{N}(i-j)},
$$

implying that $h$ and $H$ are isospectral, where $U$ is as defined in Eq. (66). It may be noted that, in general, eigenstates of $h$ and $H$ are different. However, with proper identification of physical observables in $\mathcal{H}_{\eta_{+}}$through Eq. (4), different correlation functions of the quantum systems governed by $H$ and $h$ are identical. 


\section{CONCLUSIONS \& DISCUSSIONS}

A class of pseudo-hermitian quantum systems with a pre-determined metric in the Hilbert-space has been presented. These quantum systems admit entirely real spectra. Moreover, the time-evolution is unitary with the use of the modified inner product in the Hilbert space. The general approach that has been followed in the construction of these quantum systems is the following. The basic canonical commutation relations defining these systems have been realized in terms of operators those are non-hermitian with respect to the Dirac-hermiticity condition, but, are hermitian with respect to the modified inner product in the Hilbert space involving the pre-determined metric. Consequently, appropriate combinations of these operators result in a very large number of pseudo-hermitian quantum systems. The examples considered in this paper include higher dimensional simple harmonic oscillators with complex angular frequencies, Stark effect with complex electric field, Zeeman effect with non-hermitian interaction, non-hermitian general quadratic form of $N$ boson(fermion) operators, $X X Z$ spin-chains with complex magnetic field, a non-hermitian version of Haldane-Shastry spin-chain and Lipkin-Meshkov-Glick model.

The results presented in this paper are purely mathematical. An experimental realization or verification of the predictions emanating from the study of pseudo-hermitian $/ \mathcal{P} \mathcal{T}$ symmetric quantum mechanics is desirable. Although a concrete proposal on how nonhermitian interaction of the form described in this paper could be realized experimentally is lacking, it is worth mentioning possible signatures in support/violation of $\mathcal{P} \mathcal{T}$ symmetric/pseudo-hermitian quantum physics, even within hypothetical set-ups. In this regard, the examples considered in Sec. III and time-evolution of asymmetric XXZ Hamiltonian may be promising scenarios. For example, if non-hermitian interaction of the form described in Sec. III can be produced in the laboratory with $\gamma$ being one of the externally controllable parameters, transition rate between two allowed levels may be studied for $\gamma=0$ and $\gamma \neq 0$. It may be recalled here that in the Hilbert space $\mathcal{H}_{D}, \gamma=0$ and $\gamma \neq 0$ correspond to hermitian and non-hermitian Hamiltonian, respectively. According to the prediction of 
this paper, the transition rate between any two allowed levels would be independent of $\gamma$, if nature realizes pseudo-hermitian $/ \mathcal{P} \mathcal{T}$-symmetric quantum systems.

In a similar way, the time-evolution of $H_{A}$ in $\mathcal{H}_{D}$ is expected to be non-unitary, while it is unitary in $\mathcal{H}_{\eta_{+}}$. At a purely formal level, it might seem to be a matter of choice to describe either unitary or non-unitary time-evolution by fixing an appropriate metric in the Hilbert space. However, an experimental realization of any one of these systems related to reaction-diffusion processes and Kardar-Parisi-Zhang-type growth phenomenon may give a definite answer on whether nature realizes pseudo-hermitian quantum systems or not and whether or not a more general positive-definite metric in the Hilbert space than the one

prescribed by Dirac is allowed. Any experimental result indicating the independence of different types of correlation functions on $\gamma[8]$ would garner support in favor of pseudohermitian $/ \mathcal{P} \mathcal{T}$-symmetric quantum mechanics

\section{Acknowledgments}

The Author would like to thank Andreas Fring and Mikhail S. Plyushchay for bringing to his attention the references [17] and [19], respectively.

[1] C. M. Bender, Contemp. Phys. 46, 277(2005); C. M. Bender, D. C. Brody and H. F. Jones, Am. J. Phys. 71,1095 (2003); C.M. Bender and S. Boettcher, Phys. Rev. Lett. 80, 5243(1998).

[2] A. Mostafazadeh, arXiv:0810.5643; A. Mostafazadeh, J. Math Phys. 43, 205(2002); 43, 2814(2002); 43, 3944(2002).

[3] L. Solombrino, J. Math. Phys. 43, 5439 (2002); G. Scolarici and L. Solombrino, J. Math. Phys. 44, 4450 (2003).

[4] F. G. Scholtz, H. B. Geyer and F. J. W. Hahne, Ann. Phys. 213, 74 (1992).

[5] P. Dorey, C. Dunning and R. Tateo, J. Phys. A 34, 5679(2001); J. Phys. A40, R205(2007).

[6] M. S. Swanson, Jour. Math. Phys. 45, 585 (2004). 
[7] P. K. Ghosh, J. Phys. A38, 7313 (2005); T. Deguchi and P. K. Ghosh, Phys. Rev. E 80, 021107(2009); T. Deguchi, P. K. Ghosh and K. Kudo, Phys. Rev. E 80, 026213 (2009).

[8] T. Deguchi and P. K. Ghosh, J. Phys. A42, 475208(2009).

[9] O. A. Castro-Alvaredo and A. Fring, J. Phys. A42, 465211 (2009); C. Korff, J. Phys. A41, 295206(2008); C. Korff and R. A. Weston, J. Phys. A40, 8845 (2007).

[10] C. Quesne, J. Phys. A40, F745 (2007); D. P. Musumbu, H. B. Geyer and W. D. Heiss, J. Phys. A40, F75 (2007); A. Sinha and P. Roy, J. Phys. A40, 10599 (2007).

[11] M. Znojil, Phys. Lett. A259, 220 (1999); Z. Ahmed, Phys. Lett. A282, 343 (2001).

[12] P. K. Ghosh and K.S. Gupta, Phys. Lett. A323, 29(2004); P. K. Ghosh, Eur. Phys. J. C42, $355(2005)$.

[13] A. Mostafazadeh and A. Batal, J. Phys. A37, 11645(2004); A. Mostafazadeh, J. Math. Phys. 46, 102108 (2005); C. M. Bender and B. Tan, J. Phys. A39, 1945 (2006).

[14] C. M. Bender, D. C. Brody and H. F. Jones, Phys. Rev. D70, 025001 (2004); H. F. Jones, J. Phys. A38, 1741 (2005); A. Mostafazadeh, J. Phys. A38, 6557 (2005).

[15] F. G. Scholtz and H. B. Geyer, Phys. Lett. B634, 84 (2006).

[16] A. Das and L. Greenwood, Phys. Lett. B678, 504(2009).

[17] C. F. de Morisson Faria and A. Fring, J. Phys. A39, 9269 (2006).

[18] H. F. Jones, J. Phys. A42, 135303(2009).

[19] S. M. Klishevich and M. S. Plyushchay, Nucl. Phys. B628, 217 (2002).

[20] U. Guenther and B. F. Samsonov, Phys. Rev. A 78, 042115(2008); U. Guenther and B. F. Samsonov, Phys. Rev. Lett. 101, 230404(2008).

[21] S. Albeverio, U. Guenther and S. Kuzhel, J. Phys. A42, 105205(2009).

[22] E. M. Graefe, U. Guenther, H. J. Korsch, A. E. Niederle, J. Phys. A41, 255206(2008).

[23] F. D. M. Haldane, Phys. Rev. Lett. 60, 635 (1988); B. S. Shastry, Phys. Rev. Lett. 60, 639 (1988).

[24] H. J. Lipkin, N. Meshkov and A. J. Glick, Nucl. Phys. 62, 88 (1965); ibid. 199(1965); ibid. 211(1965). 
[25] G. Dattoli, A. Renieri, A. Torre and Juan C. Gallardo, Phys. Rev. A35, 4175(1987); G. Dattoli, A. Torre, G. Reali and M. Richetta, Il Nuovo Cimento, B101, 585 (1988).

[26] A. Jannussis, Il Nuovo Cimento B 94, 29(1986);

[27] L. C. Papaloucas, Il Nuovo Cimento B 102, 315 (1988).

[28] S. Baskoutas, A. Jannussis, R. Mignani and V. Papatheou, J. Phys. A26, L819(1993).

[29] R. Santra, Phys. Rev. A74, 034701 (2006).

[30] A. Jannussis, K. Vlachos, V. Papatheou and A. Streklas, Int. J. Mod. Phys. B20, 2313 (2006).

[31] N. Hatano and D. R. Nelson, Phys. Rev. Lett. 77, 570(1997); ibid. Phys. Rev. B56, 8651 (1997).

[32] J. L. van Hemmen, Z. Physik B-Condensed Matter 38, 271 (1980).

[33] R. Orus, S. Dusuel, J. Vidal, Phys. Rev. Lett. 101, 025701 (2008); H. T. Cui, Phys. Rev. A77, 052105 (2008); S. Morrison, A. S. Parkins, Phys. Rev. Lett. 100, 040403 (2008); N. Debergh, Fl. Stancu, J. Phys. A 34, 3265(2001).

[34] E. Lieb, T. Schultz and D. Mattis, Annals of Physics 16, 407 (1961).

[35] B. M. McCoy and T. T. Wu, Il Nuovo Cimento B56, 311 (1968).

[36] H. Au-Yang and J. H. H. Perk, Adv. Stud. Pure Math 19, 57 (1989).

[37] G. Albertini, B. M. McCoy and J. H. H. Perk, Adv. Stud. Pure Math. 19, 1 (1989).

[38] F. C. Alcaraz, M. Droz, M. Henkel and V. Rittenberg, Ann. Phys. (N.Y.) 230, 250 (1994); B. Derrida, M. R. Evans, V. Hakim and V. Pasquier, J. Phys. A: Math. Gen. 26, 1493 (1993); T. Sasamoto and M. Wadati, J. Phys. A 31, 6057 (1998); T. Fukui and N. Kawakami, Nucl. Phys. B519, 715 (1998); U. Bilstein and B. Wehefritz, J. Phys. A30, 4925(1997); S. Sandow, Phys. Rev. E50, 2660 (1994); G. Albertini, S. R. Dahmen and B. Wehefritz, J. Phys. A29, L369 (1996); G. M. Schütz, Integrable Stochastic Many-body Systems, in Phase Transition and Critical Phenomena Vol. 19 Eds., C. Domb and J. L. Lebowitz, (Academic, London, 2000).

[39] von Gehlen G, J. Phys. A 24, 5371(1991); von Gehlen G, Int. J. Mod. Phys. B 8, 3507 (1994).

[40] C. N. Yang and T. D. Lee, Phys. Rev. 87, 404 (1952); T. D. Lee and C. N. Yang, Phys. Rev. 
87, 410(1952).

[41] J. L. Cardy, Phys. Rev. Lett. 54, 1354 (1985).

[42] S. Katsura, Phys. Rev. 127, 1508 (1962); ibid. 129, 2835 (1963).

[43] V. Pasquier and H. Saleur, Nucl. Phys. B330, 523 (1990).

[44] T. Inami and H. Konno, J. Phys. A27, L913 (1994); H. J. de Vega and A. Gonzales-Ruiz, J. Phys. A27, 6129 (1994).

[45] T. Deguchi, SIGMA 02, 021 (2006); J. Phys. A37, 347(2004). 